\title{
Cochrane review summary: specialised antenatal clinics for women with a multiple pregnancy for improving maternal and infant outcomes
}

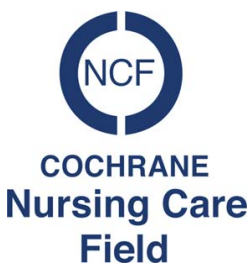

Daksha Trivedi

Senior Research Fellow, Evidence Based Practice, Centre for Research in Primary and Community Care, University of Hertfordshire, Hatfield, UK

Key words: antenatal care; infant health; maternal health; multiple pregnancy; prenatal care; twin

\section{Review question}

What are the benefits and harms of 'specialised' antenatal care for women with a multiple pregnancy compared with 'standard' antenatal care?

\section{Relevance to primary care and nursing}

The National Institute for Health and Clinical Excellence (NICE, 2011) recommends that clinical care for women with multiple pregnancies should be provided by a specialist multidisciplinary team involving specialist midwives with experience and knowledge of managing multiple pregnancies. It highlights that current evidence, based on observational studies is of low quality. This systematic review has summarised all the available evidence on specialised antenatal care for women with a multiple pregnancy for improving maternal and infant outcomes (Dodd and Crowther, 2012).

\section{Type of review}

This is a summary of a Cochrane systematic review that included one randomised controlled trial (RCT) evaluating a specialised intervention for a multiple pregnancy compared with standard care.

Correspondence to: Dr Daksha Trivedi, Senior Research Fellow, Evidence Based Practice, Centre for Research in Primary and Community Care, University of Hertfordshire, College Lane, Hatfield, AL10 9AB, UK. Email: d.trivedi@herts.ac.uk

\section{Characteristics of the evidence}

Interventions needed to be specialist antenatal care provided to women with a multiple pregnancy (as defined by trial authors) and evaluated in RCTs. Primary outcomes of interest were:

1. Perinatal death (stillbirth of one or more infants after trial entry, or death of one or more live born infants up to 28 days of age).

2. Small-for-gestational age (birth weight less than the 10th centile for gestational age).

3. Very preterm birth (birth before 34 weeks' gestation).

4. Maternal death.

Secondary outcomes included:

1. Pregnancy outcomes: antenatal complications and investigations, preterm birth (birth before 37 weeks' gestation), extremely preterm birth (birth before 28 weeks' gestation), maternal admission to intensive care unit, infection requiring intravenous antibiotics, haemorrhage requiring blood transfusion, uterine rupture, mode of birth, postnatal depression, breastfeeding.

2. Complications for infants.

3. Satisfaction.

4. Costs ('specialised' antenatal care versus 'standard' care, antenatal visits, number and length of admissions, length of maternal postnatal stay, infant hospital stay and length of stay in neonatal intensive care unit).

One UK trial was identified which included 162 women with a twin pregnancy (and 324 infants) 
randomised to receive standard antenatal care (involving consultation with the woman's general practitioner, consultant obstetrician, community midwife, antenatal education sessions and breastfeeding workshop), or specialist antenatal care (involving the standard care in addition to midwifery-led antenatal and postnatal home visits, as well as an antenatal preparation for parenting programme). They were booked for care before 20 weeks' gestation, with no known foetal anomalies. The study measured perinatal mortality as the primary outcome and caesarean birth, postnatal depression, breastfeeding, stillbirth and neonatal death as secondary outcomes.

\section{Summary of key evidence}

There were no excluded studies. The quality of the included RCT was assessed as methodologically sound. The effects of interventions were analysed according to primary and secondary outcomes. Data were pooled using risk ratios (RR) with $95 \%$ confidence intervals (CI). The numbers are shown in parenthesis.

\section{Primary outcomes}

One primary outcome prespecified in the review was reported. There were no significant differences between specialised antenatal care and standard care for perinatal mortality (RR 1.02; 95\% CI 0.26-4.03; one study, $n=324$ infants). Data on small-for-gestational age, very preterm birth or maternal death were not reported.

\section{Secondary outcomes}

Specialised antenatal care provided to women significantly increased the likelihood of requiring a caesarean birth (RR 1.38; 95\% CI 1.06-1.81; one study, $n=162$ women) compared with standard antenatal care. No significant differences were reported between the two groups for: postnatal depression (RR 0.48; 95\% CI 0.19-1.20; one study, $n=133$ women) and breastfeeding at six months (RR 0.63; 95\% CI 0.24-1.68; one study, $n=123$ women). There were no significant effects on stillbirth (RR 0.68; 95\% CI 0.12-4.04; one study, $n=324$ infants) or neonatal death (RR 2.05 ; $95 \%$ CI $0.19-22.39$; one study, $n=324$ infants) which were not prespecified in the review protocol.

\section{Implications for practice}

Only one RCT evaluating specialist antenatal care was identified. There is currently insufficient evidence to establish the effectiveness of 'specialised' antenatal clinics for women with a multiple pregnancy compared with 'standard' antenatal care.

\section{Implications for research}

High quality well-designed trials, appropriately powered and measuring relevant maternal and infant health outcomes are required to establish the effectiveness and the value of 'specialised' multiple pregnancy clinics.

\section{Acknowledgement}

The author is a member of the Cochrane Nursing Care Field (CNCF).

\section{Financial Support}

This research received no specific grant from any funding agency, commercial or not-for-profit sectors.

\section{Statement of Interest}

None.

\section{Ethical Standards}

Not applicable. This is a summary based on secondary research and is not dealing with animals.

\section{References}

Dodd, J.M. and Crowther, C.A. Specialised antenatal clinics for women with a multiple pregnancy for improving maternal and infant outcomes. Cochrane Database of Systematic Reviews 2012: Art. No.: CD005300. doi:10.1002/14651858. CD005300.pub3.

National Institute for Health and Clinical Excellence (NICE). 2011. Clinical guideline 129, multiple pregnancy: The management of twin and triplet pregnancies in the antenatal period. Manchester: NICE. Retrieved 16 August 2013 from http:// www.nice.org.uk/nicemedia/live/13571/56422/56422.pdf 